\title{
Superconvergent Renormalization and Tensor Approximation
}

\author{
Edwards, Michael G. \\ University of Texas at Austin, Austin Texas 78712 USA
}

Paper Presented at the 5th European Conference on the Mathematics of Oil Recovery, Leoben Austria, 3-6 Sept. 1996

\section{INTRODUCTION}

A real reservoir can have a complex geometric and geologic description and use of a non-orthogonal grid allows great flexibility and realism in the modeling process. A full tensor velocity field is essential when using non-orthogonal grids, e.g.[1] modeling cross bedding [2] and including upscaled tensor permeability effects [3]. Support of the standard scheme has to increase in such cases (to typically 9 nodes in 2-D); a standard five point scheme has an $O(1)$ error in flux on a nonorthogonal grid [4] (an example of the consequence is shown in the results section of Fig 6). Permeabilities can vary by orders of magnitude from cell to cell and it is essential that flux continuity (inherent in the standard scheme) be maintained in departing from a five point ( 7 in 3-D) scheme.

A general cell centered flux continuous scheme comes at a price. In essence besides increasing the scheme support to 9 nodes (19 or 27 in 3-D), interface pressures have to be introduced as part of the discretization. They can be eliminated algebraically $[4,5]$ or included as additional degrees of freedom [6]. The latter approach involves a larger discretization matrix. While the former approach has a relatively involved implementation and generally yields a non-symmetric discrete matrix [4], equality between the number of degrees of freedom and the number of cells is maintained. This is discussed below.

In this paper it is demonstrated that the scheme of [4] can be employed as an improved renormalization operator for diagonal and full tensors. Since an application of a renormalization operator on a $2 \times 2$ cell formation yields an effective permeability at the formation center, this provides a natural means of mapping cell centered permeabilities to cell vertices. This shift permits a transition to a general finite volume framework [7], with pressures located at the cell centers discrete symmetric positive definite (9 point in 2-D 19 or 27 in 3-D) schemes are constructed. Properties of the framework are discussed and a comparison is made between results obtained by the flux continuous scheme of [4] and the finite volume scheme with renormalized coefficients.

\section{THE PROBLEM POSED}

The problem is to find the pressure $\phi$ satisfying

$$
\int_{\Omega}-\nabla \cdot \mathbf{K}(\mathrm{x}, \mathrm{y}) \nabla \phi \mathrm{d} \tau=\int_{\Omega} \mathrm{qd} \tau=\mathrm{m}
$$

over an arbitrary domain $\Omega$, subject to suitable (Neumann/Dirichlet) boundary conditions on boundary $\partial \Omega$. The right hand side term $\mathrm{m}$ represents a flow rate, and $\nabla=\left(\partial_{x}, \partial_{y}\right)$. Matrix $\mathbf{K}$ can be a diagonal or full cartesian tensor with general form

$$
\mathbf{K}=\left(\begin{array}{ll}
K_{\alpha \alpha} & K_{\alpha \beta} \\
K_{\beta \alpha} & K_{\beta \beta}
\end{array}\right)
$$

The full tensor pressure equation is assumed to be "elliptic" such that

$$
\left(\frac{K_{\alpha \beta}+K_{\beta \alpha}}{2}\right)^{2} \leq K_{\alpha \alpha} K_{\beta \beta}
$$

The tensor can be discontinuous across internal boundaries of $\Omega$. For reservoir simulation, Neumann boundary conditions on $\partial \Omega$ require zero flux such that $(K \nabla \phi) . \hat{\mathbf{n}}=0$, where $\hat{n}$ is the outward unit normal vector to $\partial \Omega$.

\section{GENERAL TENSOR EQUATION}

The pressure equation is defined above with respect to the "physical" tensor in the initial classical cartesian coordinate system. Now we proceed to a general curvilinear coordinate system that is defined via a mapping e.g. [1] with respect to a uniform dimensionless cartesian $(\xi, \eta)$ coordinate system. Choosing $\Omega_{p}$ to represent an arbitrary control volume comprised of surfaces that are tangential to constant $(\xi, \eta)$ respectively, equation 2.1 is integrated over $\Omega_{\mathrm{p}}$ via the Gauss flux theorem to yield 


$$
\oint_{\partial \Omega_{\mathrm{p}}}(K \nabla \phi) \cdot \hat{n} \mathrm{ds}=\mathrm{m}
$$

where $\partial \Omega_{p}$ is the boundary of $\Omega_{p}$ and $\hat{n}$ is the unit outward normal. Spatial derivatives are computed using

$$
\phi_{\mathrm{x}}=\mathrm{J}(\phi, \mathrm{y}) / \mathrm{J}(\mathrm{x}, \mathrm{y}) \quad \phi_{\mathrm{y}}=\mathrm{J}(\mathrm{x}, \phi) / \mathrm{J}(\mathrm{x}, \mathrm{y})
$$

where $J(x, y)=x_{\xi} y_{\eta}-x_{\eta} y_{\xi}$. Resolving the $x, y$ components of velocity along the unit normals to the curvilinear coordinates $(\xi, \eta)$ ( e.g for $\xi=$ constant $\hat{\mathrm{nds}}=\left(\mathrm{y}_{\eta},-\mathrm{x}_{\eta}\right) \mathrm{d \eta}$ gives rise to the general tensor flux components

$$
\begin{aligned}
& F=-\int\left(T_{a a} \phi_{\xi}+T_{a b} \phi_{\eta}\right) d \eta, \\
& G=-\int\left(T_{b a} \phi_{\xi}+T_{b b} \phi_{\eta}\right) d \xi
\end{aligned}
$$

where the general tensors are defined by

$$
\begin{aligned}
\mathrm{T}_{\mathrm{aa}}= & \left(\mathrm{K}_{\alpha \alpha} \mathrm{y}_{\eta}^{2}+\mathrm{K}_{\beta \beta} \mathrm{x}_{\eta}^{2}-\left(\mathrm{K}_{\alpha \beta}+\mathrm{K}_{\beta \alpha}\right) \mathrm{x}_{\eta} \mathrm{y}_{\eta}\right) / \mathrm{J} \\
\mathrm{T}_{\mathrm{ab}}= & \left(\mathrm{K}_{\alpha \beta} \mathrm{x}_{\xi} \mathrm{y}_{\eta}+\mathrm{K}_{\beta \alpha} \mathrm{x}_{\eta} \mathrm{y}_{\xi}\right. \\
& \left.-\left(\mathrm{K}_{\alpha \alpha} \mathrm{y}_{\xi} \mathrm{y}_{\eta}+\mathrm{K}_{\beta \beta} \mathrm{x}_{\xi} \mathrm{x}_{\eta}\right)\right) / \mathrm{J} \\
\mathrm{T}_{\mathrm{ba}}= & \left(\mathrm{K}_{\alpha \beta} \mathrm{y}_{\xi} \mathrm{x}_{\eta}+\mathrm{K}_{\beta \alpha} \mathrm{y}_{\eta} \mathrm{x}_{\xi}\right. \\
& \left.-\left(\mathrm{K}_{\alpha \alpha} \mathrm{y}_{\xi} \mathrm{y}_{\eta}+\mathrm{K}_{\beta \beta} \mathrm{x}_{\xi} \mathrm{x}_{\eta}\right)\right) / \mathrm{J} \\
\mathrm{T}_{\mathrm{bb}}= & \left(\mathrm{K}_{\alpha \alpha} \mathrm{y}_{\xi}^{2}+\mathrm{K}_{\beta \beta} \mathrm{x}_{\xi}^{2}-\left(\mathrm{K}_{\alpha \beta}+\mathrm{K}_{\beta \alpha}\right) \mathrm{x}_{\xi} \mathrm{y}_{\xi}\right) / \mathrm{J}
\end{aligned}
$$

and the closed integral can be written as

$$
\iint_{\Omega_{p}} \frac{\left(\partial_{\xi} F+\partial_{\eta} G\right)}{J} J d \xi d \eta=\Delta_{\xi} F+\Delta_{\eta} G=m
$$

where e.g. $\Delta_{\xi} F$ is the difference in net flux with respect to $\xi$. Thus any scheme applicable to a full tensor also applies to non-orthogonal grids.

Note that $T_{a a}, T_{b b} \geq 0$ provided Eq 2.2 holds, and Eq 3.4 exposes the idea of a "K-orthogonal" grid system, if a grid is generated such that $T_{a b}=T_{b a}=0$ then in principle, full tensor effects can be included while fluxes need only be a function of the usual difference in adjacent pressures with respect to the K-orthogonal grid system. This approach is under investigation $[8,9]$

\section{FLUX CONTINUOUS APPROXIMATIONS}

While the focus of this paper is on cell centered schemes where pressure and permeability are located at the centers of the grid cells, it will be instructive to draw comparisons with cell vertex based approximations where pressures are located at the vertices of the grid cells while permeability remains cell centered. We begin with the classical cell centered approximation in one dimension, where $\mathrm{Eq} 2.1$ reduces to

$$
-\left(K(x) \phi_{x}\right)_{x}=q
$$

and $\mathrm{K}$ can be cell-wise discontinuous. Integration of the equation Eq 3.1 over the cell $\mathrm{i}$ (referring to Fig 1 ) results in the discrete difference of fluxes

$$
f_{i+1 / 2}-f_{i-1 / 2}=q \Delta x=m
$$

where $m$ is a flow rate, $f_{i+1 / 2}=-K \partial \phi / \partial x$ and the derivative remains to be defined. At this stage if the coefficient $\mathrm{K}$ is sufficiently smoothly varying it is possible to use linear interpolation between the centers of cells $i$ and $i+1$ and define the flux by

$$
\mathrm{f}_{\mathrm{i}+1 / 2}=-\mathrm{K}_{\mathrm{i}+1 / 2}\left(\phi_{\mathrm{i}+1}-\phi_{\mathrm{i}}\right) / \Delta \mathrm{x}
$$

where $K_{i+1 / 2}$ is a suitable average of the adjacent cell centered permeabilities. However if $\mathrm{K}$ is discontinuous then since flux and pressure are continuous the pressure gradient is discontinuous and linear interpolation is not valid across cell faces. The standard solution to this problem in one dimension is described in [10]. Continuous pressure and flux are incorporated in the cell centered approximation by introducing a mean pressure $\phi_{\mathrm{f}}$ at a cell face dividing neighbouring cells Fig 1 . Equating the resulting one sided flux approximations at the cell face results in

$$
-\mathrm{k}_{\mathrm{r}}\left(\phi_{\mathrm{r}}-\phi_{\mathrm{f}}\right) / \Delta \mathrm{x}_{\mathrm{r}}=-\mathrm{k}_{\mathrm{l}}\left(\phi_{\mathrm{f}}-\phi_{1}\right) / \Delta \mathrm{x}_{1}
$$

which ensures flux continuity. From Eq 4.3 the cell face pressure is given by

$$
\begin{aligned}
& \phi_{\mathrm{f}}=\left(\phi_{1} \mathrm{k}_{\mathrm{l}} / \Delta \mathrm{x}_{1}+\phi_{\mathrm{r}} \mathrm{k}_{\mathrm{r}} / \Delta \mathrm{x}_{\mathrm{r}}\right) \\
& /\left(\mathrm{k}_{\mathrm{l}} / \Delta \mathrm{x}_{1}+\mathrm{k}_{\mathrm{r}} / \Delta \mathrm{x}_{\mathrm{r}}\right)
\end{aligned}
$$

which is back-substituted into the discrete flux (4.3) to yield the classical cell face flux approximation

$\mathrm{F}=-2 \mathrm{k}_{\mathrm{r}} \mathrm{k}_{1}\left(\phi_{\mathrm{r}}-\phi_{1}\right) /\left(\mathrm{k}_{\mathrm{r}} \Delta \mathrm{x}_{1}+\mathrm{k}_{1} \Delta \mathrm{x}_{\mathrm{r}}\right)$

We can interpret $\mathrm{Eq} 4.3$ as in effect defining a local upscaling problem for 2 half cells where $\phi_{1}, \phi_{r}$ supply the boundary conditions and Eq 4.5 defines the "coarse" flux, the harmonic mean is the effective permeability flux coefficient of the "single block" that replaces the two half cells and therefore interpolation between 1 and $r$ is now valid, justifying the use of the central difference in potential across the "block". Since Eq 4.5 holds for any $\phi_{1}, \phi_{\mathrm{r}}$ then flux and pressure continuity have been obtained in a locally exact homogenized sense. Perhaps the ultimate illustration of the value of the harmonic mean is seen in the case where one of two neighbouring cells has zero conductance, the homogenized flux is zero (as required by flux continuity) while for a non-zero pressure drop, the arithmetic mean would sustain a nonzero flux yielding in an $\mathrm{O}(1)$ flux error (cf results section Fig 8b). 
These observations also tell us that if we want flux continuity without homogenization, then for a cell centered scheme we must include an additional set of pressures located at the cell faces together with the cell centered pressures. In general for dimension $D$, this leads to $\mathrm{D}+1$ times as many degrees of freedom. A naturally flux continous scheme can be obtained without increasing the number of degrees of freedom by using a cell vertex based approximation which generalises to higher dimensions with flux continuity across control volume interfaces [7]. Another possibility is to move to cell face pressure based schemes [2].

Returning to the classical cell centered formalism and continuity by exact homogenization, it is important to notice that in stating Eq 4.3 a locally continuous piecewise linear variation in pressure is assumed over each half cell interval between the cell center and cell face pressures e.g.

$$
\phi=(1-\xi) \phi_{\mathrm{L}}+\xi \phi_{\mathrm{F}} \quad \mathrm{x}_{\mathrm{L}} \leq \mathrm{x} \leq \mathrm{x}_{\mathrm{F}}
$$

for the left half cell, and produces a piecewise constant flux over each half cell interval. The support and basis functions are shown in Fig 1. The generalization of this observation provides the basis of the flux continuous locally conservative scheme presented in [4] and the scheme derivation is summarized below.

In two dimensions the domain $\Omega$ is subdivided into quadrilateral cells $\Omega_{p} p=1 \ldots N$, and approximate pressures $\phi_{i, j}$ are sought at cell centers. Each quadrilateral is mapped to a unit cell through the isoparametric map (with local node numbering 1..4)

$$
\mathbf{r}=(1-\tilde{\xi})(1-\tilde{\eta}) \mathbf{r}_{1}+\tilde{\xi}(1-\tilde{\eta}) \mathbf{r}_{2}+\tilde{\xi} \tilde{\eta} \mathbf{r}_{3}+(1-\tilde{\xi}) \tilde{\eta} \mathbf{r}_{4} 4.6
$$

where $\mathbf{r}=(\mathrm{x}, \mathrm{y})$ and $0 \leq \tilde{\xi}, \tilde{\eta} \leq 1$ per cell, while each domain maps [1] to a unit square Fig 2. The two dimensional equivalent of $\mathrm{Eq} 4.3$ is obtained by introducing cell face pressures $\left(\phi_{\mathrm{N}}, \phi_{\mathrm{S}}, \phi_{\mathrm{E}}, \phi_{\mathrm{W}}\right)$ as in Fig 3 , and a local triangular support is imposed within each quarter of a unit cell as in Fig 3. Pressure $\phi$ and the local cell coordinates assume a piecewise linear variation over each triangle, for example over the triangle of cell 3

$$
\begin{aligned}
& \phi=(1-u-v) \phi_{\mathrm{E}}+u \phi_{3}+v \phi_{\mathrm{N}} \\
& \tilde{\xi}=(1-u-v) \tilde{\xi}_{\mathrm{E}}+u \tilde{\xi}_{3}+v \tilde{\xi}_{\mathrm{N}} \\
& \tilde{\eta}=(1-u-v) \tilde{\eta}_{\mathrm{E}}+u \tilde{\eta}_{3}+v \tilde{\eta}_{\mathrm{N}}
\end{aligned}
$$

where $(u, v)$ are area coordinates [11], $\left(\tilde{\xi}_{\mathrm{E}}, \tilde{\eta}_{\mathrm{E}}\right), \quad\left(\tilde{\xi}_{\mathrm{N}}, \tilde{\eta}_{\mathrm{N}}\right)$ are local continuity coordinates and pressure is globally continuous. Differentiation of Eq 4.7 leads to well known piecewise constant gradients on triangles [11]

$$
\phi_{\tilde{\xi}}=\mathrm{J}(\phi, \tilde{\eta}) / \mathrm{J}(\tilde{\xi}, \tilde{\eta}), \quad \phi_{\tilde{\eta}}=\mathrm{J}(\tilde{\xi}, \phi) / \mathrm{J}(\tilde{\xi}, \tilde{\eta})
$$

where $J(\tilde{\xi}, \tilde{\eta})=\tilde{\xi}_{u} \tilde{\eta}_{v}-\tilde{\xi}_{v} \tilde{\eta}_{u}$ is the Jacobian.
In general flux continuity is imposed on the interfaces at positions of contineus pressure (N,S,E,W) of cells 1 to 4 sharing a common vertex (Fig 3) through

$$
\begin{aligned}
& \left.\left(T_{\mathrm{aa}} \phi_{\tilde{\xi}}+\mathrm{T}_{\mathrm{ab}} \phi_{\tilde{\eta}}\right)\right|_{\mathrm{S}} ^{1}=\left.\left(\mathrm{T}_{\mathrm{aa}} \phi_{\tilde{\xi}}+\mathrm{T}_{\mathrm{ab}} \phi_{\tilde{\eta}}\right)\right|_{\mathrm{S}} ^{2} \\
& \left.\left(\mathrm{~T}_{\mathrm{aa}} \phi_{\tilde{\xi}}+\mathrm{T}_{\mathrm{ab}} \phi_{\tilde{\eta}}\right)\right|_{\mathrm{N}} ^{3}=\left.\left(\mathrm{T}_{\mathrm{aa}} \phi_{\tilde{\xi}}+\mathrm{T}_{\mathrm{ab}} \phi_{\tilde{\eta}}\right)\right|_{N} ^{4} \\
& \left.\left(\mathrm{~T}_{\mathrm{ba}} \phi_{\bar{\xi}}+\mathrm{T}_{\mathrm{bb}} \phi_{\tilde{\eta}}\right)\right|_{\mathrm{E}} ^{2}=\left.\left(\mathrm{T}_{\mathrm{ba}} \phi_{\tilde{\xi}}+\mathrm{T}_{\mathrm{bb}} \phi_{\tilde{\eta}}\right)\right|_{\mathrm{E}} ^{3} \\
& \left.\left(\mathrm{~T}_{\mathrm{ba}} \phi_{\tilde{\xi}}+\mathrm{T}_{\mathrm{bb}} \phi_{\tilde{\eta}}\right)\right|_{\mathrm{W}} ^{1}=\left.\left(\mathrm{T}_{\mathrm{ba}} \phi_{\tilde{\xi}}+\mathrm{T}_{\mathrm{bb}} \phi_{\tilde{\eta}}\right)\right|_{\mathrm{W}} ^{4}
\end{aligned}
$$

yielding a linear system of equations for the interface pressures in terms of the four adjacent cell centered pressures. As in one dimension the cell face pressures can either be determined algebraically in terms of the cell centered pressures or the continuity conditions can be added to the pressure equation matrix in order to determine the additional degrees of freedom. Here we pursue the former option and repeat the procedure described in [4] and express each group of 4 interface pressures in terms of the 4 cell centered pressures whose union contains the continuity positions. Finally by using Eq's 4.8 and 4.9 continuous full tensor fluxes (in the exact homogenized sense of the harmonic mean described above) are obtained at each of the positions of continuity, and each flux is a linear function of the 4 cell centered pressures. Assembly of fluxes for a cell yields a 9 point scheme.

\section{Family of Schemes}

This procedure yields a whole family of schemes (for a full or diagonal tensor) defined by the free parameters that are the positions of flux continuity. For example in the limiting case, if we choose $\bar{\xi}_{\mathrm{E}}=\bar{\eta}_{\mathrm{N}}=1 / 2$ then for a diagonal tensor the scheme reduces to the standard 5 point scheme with coefficients defined by the harmonic mean of adjacent permeabilities, and has a point-wise continuous support comprised of right-angled triangles. Alternatively choosing $\bar{\xi}_{E}=\bar{\eta}_{N}=1 / 4$, (point-wise triangular continuity of Fig 3) yields a well known 9 point discretization in the case of the Laplacian operator [4] that has a 6th order accurate truncation error for constant coefficients [12]. The truncation error of the scheme with $\tilde{\xi}=1 / 4$ (for constant coefficients and exact metrics) is [13]

$-d \xi^{2} J^{-1} \frac{\left(\phi_{\xi \xi \xi \eta}+\phi_{\eta \eta \eta \xi}\right)\left(T_{a b}+T_{b a}\right)}{12}+O\left(d \xi^{4}\right)$

and thus even for an anisotropic diagonal cartesian tensor with constant coefficients the scheme undergoes super convergence for $\tilde{\xi}=1 / 4$ and is 4th order accurate [13] and is 2nd order otherwise. In particular for an arbitrary grid aspect ratio $A_{r}=Y / X$ where $x=X \xi$ and $y=Y \eta$ the equation reduces to 
$K_{\alpha \alpha} \frac{\phi_{\xi \xi}}{X^{2}}+K_{\beta \beta} \frac{\phi_{\eta \eta}}{Y^{2}}=0$

for constant coefficients with general anisotropic ratio

$$
\mathrm{R}=\mathrm{X}^{2} \mathrm{~K}_{\beta \beta} /\left(\mathrm{Y}^{2} \mathrm{~K}_{\alpha \alpha}\right)
$$

and by Eq's $4.11,4.12$ and 4.13, fourth order accuracy holds for any anisotropy ratio and thus for any grid aspect ratio and any permeability ratio.

\section{RENORMALIZATION}

Renormalization is a practical technique for upscaling rock properties from the fine scale to the grid block in reservoir simulation [14]. The grid block or domain to be upscaled is decomposed into a set of hierarchical subdomains $\omega_{d} d$ $=1, \mathrm{~N}$, so that at any given grid level each subdomain $\omega_{\mathrm{d}}$ contains mxm cells (in 2-D) and each of these cells are subdomains at the next grid level also containing mxm cells. The renormalization technique proceeds by performing independent local simulations on each subdomain. The effective permeability of $\omega_{\mathrm{d}}$ is defined such that flux through the outlet boundary, computed on the local fine grid (typically subject to no-flow top and bottom boundary conditions), is equal to the product of the effective permeability and the globally imposed pressure gradient across $\omega_{\mathrm{d}}$. The process is repeated in a hierarchical fashion. Since the $2 \times 2$ cell renormalization scheme of [14] for a diagonal tensor can be derived by performing a local single phase simulation on a $2 \times 2$ grid using the classical 5 point scheme with coefficients defined by the harmonic mean of adjacent permeabilities to ensure continuity in pressure and flux, then the above family of flux continuous schemes offer a natural generalization for both diagonal tensor 9 point schemes and full tensor schemes to renormalization.

Since we wish to handle both diagonal and full tensor upscaling the $2 \times 2$ upscaling problem is solved using the above scheme with periodic boundary conditions as described in [3] and (in theory) yields a symmetric tensor. In this case the interface fluxes and pressures are forced to be periodic on the top and bottom boundaries of the domain while a global pressure gradient is imposed across the domain. This yields $\mathrm{Kxx}, \mathrm{Kxy}$, then the boundary conditions are rotated in the usual way to find Kyx, Kyy. Note that by using the above general tensor formulation (Eq 3.4) upscaling on non-orthogonal grids is naturally incorporated and a general tensor matrix $\mathbf{T}_{\text {eff }}$ is produced. Some $2 \times 2$ upscaling examples are considered below.

The $2 \times 2$ renormalization scheme of [14] is labelled "standard", the new $2 \times 2$ renormalization scheme is labelled "9 point" and fine grid simulation results are presented in tables.

The $2 \times 2$ cell domain is numbered anticlockwise. The first two problems have isotropic permeability fields with

(a) $\mathrm{K} 1, \ldots \mathrm{K} 4=0.01,1,0.1,100$.

$\begin{array}{ll}\text { Level } & \text { Keff } \\ 64 \times 64 & 0.284\end{array}$

$128 \times 128 \quad 0.298$

Standard Keff $=0.13 \quad ; \quad 9$ point Keff $=0.304$

(b) $\mathrm{K} 1, \ldots \mathrm{K} 4=1,9 \times 10^{5}, 10,800$

$\begin{array}{ll}\text { Level } & \text { Keff } \\ 64 \times 64 & 33.12 \\ 128 \times 128 & 40.8\end{array}$

Standard Keff $=14.44 ; \quad 9$ point $\quad$ Keff $=36.48$

the excellent agreement between the $2 \times 2$ cell 9 point simulations and the fine grid 5 point simulations is a demonstration of super-convergence of flux, especially when compared to the results obtained by the standard scheme of [14] and is mainly attributed to the extra sensitivity of a 9 point scheme to cross flow. However the results are both boundary condition dependent as well as discretization dependent as can be seen by using $\bar{\xi}=1 / 2$ which returns the scheme to the standard 5 point operator (with periodic boundary conditions) and yields a flux of 0.19 for case (a).

Finally upscaling for a strongly anisotropic case is considered where $\mathrm{Ky}=100 \mathrm{Kx}$ and

$\mathrm{Kx} 1, \ldots \mathrm{Kx} 4=0.01,1,0.1,100$.

Level Keff

$64 \times 64 \quad 0.79$

Standard Keff $=0.198 ; \quad 9$ point $\quad$ Keff $=0.38$

(By Eq 4.13 this problem (and results) is identical to that of an isotropic field with grid aspect ratio $\mathrm{Y} / \mathrm{X}=1 / 10$.) In this case the keff is a poor estimate, although the improvement is still significant compared to the standard star triangle approach. However by choosing the continuity coordinates to increase the effect of cross flow, i.e. with reduced $\bar{\xi}$, (resulting in a modified quadrature) yields 9 point Keff $=0.77$.

\section{POSITIVE DEFINITE SCHEMES}

In the general case the above scheme [4] has a nonsymmetric discrete matrix. A framework for a (locally conservative) finite volume discretization that embraces all classical 9 point scheme flux weightings, and that maintains a symmetric positive definite discrete matrix for a full tensor for all choices of quadrature subject to discrete ellipticity per cell c.f Eq 2.2, was presented in [7] together with conditions for diagonal dominance of the scheme and thus a discrete maximum principle. The remainder of this paper is concerned with an appropriate definition of a cell centered scheme within the latter framework. Let us temporarily delay the definition of the discretization with respect to the grid. Initially the discretization is defined with respect to the union of 4 elements where each element is defined by the union of four nodes, for example element 3 Fig $4 \mathrm{a}$ is centered upon the midpoint $(i+1 / 2, j+1 / 2)$ and is comprised of the 4 nodes at the corners of the element, with indices $(i, j),(i+1, j)$, 
$(i+1, j+1),(i, j+1)$ (elements and cells are mapped to unit squares via $E q$ 4.6). Approximate pressures $\phi_{i, j}$ and coefficients $\mathbf{K}_{\mathrm{i}+1 / 2, j+1 / 2}$ are located at the (corner) nodes and centers of the elements respectively. The element-wise description enables the scheme to be viewed with respect to a cell vertex or cell centered pressure approximation. If the element coincides with a grid cell then the nodes coincide with the cell vertices and the scheme is cell vertex based. If the nodes coincide with the centers of 4 adjacent grid cells then the "element" is centered upon the common grid vertex and scheme is cell centered. A control volume (shaded fig 4 ) is associated with each node and has a surface defined by a closed contour that connects the surrounding element centers together via straight lines Fig 4 as in [7].

Permeabilities $\mathbf{K}_{\mathrm{i}+1 / 2, j+1 / 2}$ are assumed to be piecewise constant over each element, enabling pressure to be approximated via polynomials of arbitrary order over each element, and flux and pressure continuity across the control volume interfaces follows immediately. In this scheme $(x, y)$ have the quadrilateral cell-wise variation defined by $\mathrm{Eq} \mathrm{4.6,} \mathrm{while} \mathrm{the} \mathrm{pressure} \mathrm{assumes} \mathrm{an}$ isoparametric bilinear variation over each element $c . f$. Eq 4.6 , replace $\mathbf{r}$ by $\phi$ for pressure where $0 \leq \tilde{\xi}, \tilde{\eta} \leq 1$ and $(1, \ldots 4)$ refers to local node numbering, and local $(\tilde{\xi}, \tilde{\eta})$ derivatives of $\mathbf{r}$ and $\phi$ are obtained by differentiation e.g.

$$
\begin{aligned}
& \phi_{\tilde{\xi}}=(1-\tilde{\eta})\left(\phi_{2}-\phi_{1}\right)+\tilde{\eta}\left(\phi_{3}-\phi_{4}\right) \\
& \phi_{\tilde{\eta}}=(1-\tilde{\xi})\left(\phi_{4}-\phi_{1}\right)+\tilde{\xi}\left(\phi_{3}-\phi_{2}\right)
\end{aligned}
$$

In defining the control volumes each element is in effect, divided into four local quadrants (one per node), and each quadrant belongs to the control volume of its attached node. The scheme can be assembled and analysed elementwise as described in [7]. Discrete fluxes are derived by integrating Eq 3.3 over the surface of the control volume Fig4 which is performed piecewise along each control volume boundary segment with respect to the local element coordinates. For a cell centered scheme, limits of integration are between cell face midpoints and cell vertices as indicated in fig $4 c$, and correspond to half cell intervals in the $\xi, \eta$ coordinate system.

$$
\begin{aligned}
& F_{S}=-\int_{0}^{1 / 2}\left(T_{a a} \phi_{\tilde{\xi}}+T_{a b} \phi_{\tilde{\eta}}\right) d \tilde{\eta} \\
& F_{N}=-\int_{1 / 2}^{1}\left(T_{a a} \phi_{\tilde{\xi}}+T_{a b} \phi_{\tilde{\eta}}\right) d \tilde{\eta} \\
& F_{E}=-\int_{1 / 2}^{1}\left(T_{b a} \phi_{\tilde{\xi}}+T_{b b} \phi_{\tilde{\eta}}\right) d \tilde{\xi} \\
& F_{W}=-\int_{0}^{1 / 2}\left(T_{b a} \phi_{\tilde{\xi}}+T_{b b} \phi_{\tilde{\eta}}\right) d \tilde{\xi}
\end{aligned}
$$

The element-wise continuous fluxes are now distributed to the element corner nodes to assemble the locally conservative scheme.

At this stage there is a variety of choices that can be made in terms of quadrature for integration. Since the transform derivatives are also functions of $(\tilde{\xi}, \tilde{\eta})$ they could be integrated, however they are naturally defined at the cell centers in terms of the geometry since they can then be included in the general piecewise constant cell centered tensor coefficients for each cell.

Direct integration of Eq 6.2 over each half interval leads to a flux weighting $(1,6,1) / 8$. However a more general flux is defined by retaining $(\tilde{\xi}, \tilde{\eta})$ as degrees of freedom allowing flexibility in the choice of quadrature [7]. With respect to global $(i, j)$ numbering of the nodes the discrete scheme can be written as

$$
\begin{aligned}
& \Delta_{\xi_{-}}\left(F_{S_{i+1 / 2, j+1 / 2}}+F_{N_{i+1 / 2, j-1 / 2}}\right)+ \\
& \Delta_{\eta_{-}}\left(F_{W_{i+1 / 2, j+1 / 2}}+F_{E_{i-1 / 2, j+1 / 2}}\right)=m_{i, j}
\end{aligned}
$$

where $\Delta_{\xi_{-}}$is a backward difference operator on $i+1 / 2$ and for element $(i+1 / 2 . j+1 / 2)$ the local node numbers $[1,2,3,4]$ correspond to $[(i, j),(i+1, j),(i+1, j+1),(i, j+1)]$ and for example

$$
\begin{aligned}
& F_{S_{i+1 / 2, j+1 / 2}}=-\left[T _ { a _ { i + 1 / 2 , j + 1 / 2 } } \left((1-\tilde{\eta})\left(\phi_{i+1, j}-\phi_{i, j}\right)\right.\right. \\
& \left.+\tilde{\eta}\left(\phi_{i+1, j+1}-\phi_{i, j+1}\right)\right) \\
& \left.+T_{a b i+1 / 2, j+1 / 2} \frac{\left(\phi_{i, j+1}-\phi_{i, j}+\phi_{i+1, j+1}-\phi_{i+1, j}\right)}{2}\right] \frac{1}{2}
\end{aligned}
$$

where the general cell centered tensor coefficients are defined via $\mathrm{Eq} 3.4$ and are functions of the discrete cell centered permeabilities e.g $\mathrm{K}_{\alpha \alpha_{i+1 / 2, j+1 / 2}}$, and transform derivatives, where for example

$$
x_{\xi_{i+1 / 2, j+1 / 2}}=\frac{\left(\left(x_{i+1, j}-x_{i, j}\right)+\left(x_{i+1, j+1}-x_{i, j+1}\right)\right)}{4} 6.5
$$

For a discrete elliptic symmetric tensor, the discrete matrix is symmetric positive definite for all quadrature points $\tilde{\eta}$ such that $0 \leq \tilde{\eta}<1 / 2$ with conditional diagonal dominance for $\tilde{\eta}>0[15,7]$. The four control volume quadrant fluxes $\mathrm{F}_{\mathrm{S}}, \mathrm{F}_{\mathrm{N}}, \mathrm{F}_{\mathrm{E}}, \mathrm{F}_{\mathrm{W}}$ are defined in [7].

\section{CELL VERTEX - CELL CENTERED}

Cell Vertex : Choosing the element to coincide with a grid cell (cell vertex scheme) the physical permeability is already defined at the cell center and Eq's $3.4,6.5$ provide the metrics that exactly correspond to the control volume surface Fig $4 \mathrm{a}$ and flux is continuous across the control volume interface. A quadrature with $\tilde{\eta}=1 / 3$ defines a flux weighting $(1,4,1) / 6$ that corresponds to "Simpsons rule" weighting, in this case the discretization is identical to the standard Galerkin finite element scheme that results from using piecewise bilinear basis functions with general tensor coefficients that are piecewise constant over each cell [15] (and [16] where quadratures and effects on grid orientation are presented). Therefore convergence of this scheme follows directly from classical finite element convergence analysis, now with respect to the $(\tilde{\xi}, \tilde{\eta})$ transform space.

Cell Centered : From the above discussion it is clear that the scheme maintains all of the above properties when choosing the points to coincide with the cell centers, however to complete the dual definition of the scheme, 
"element" permeabilities must now be defined at the vertices. One possibility is to generate permeabilities at grid vertices (vertex injection) instead of at cell centers as in current practice, however this works against the basis of the generated grid. If permeability is cell centered then a suitable mapping is required to define the cell vertex permeability field. The above tensor renormalization scheme has been demonstrated to provide an accurate effective tensor permeability mapping and nonorthogonal grid effects are included via the above general tensor $\mathbf{T}_{\text {eff }}$. Therefore the alternative cell centered scheme proposed here utilizes the framework presented in [7] and defined here with respect to elements with corner nodes that coincide with the cell centers of an arbitrary nonorthogonal grid, and with vertex centered element permeabilities defined by the renormalization scheme outlined above.

For given coefficients, the choice $\tilde{\eta}=0$ corresponds to the 9 point scheme derived from the mixed method [6], where convergence is proved. This method is used with 19 nodes for 3-D non-orthogonal multiblock grids [17]. In this case the pressure gradients aligned with the diagonal tensor components are only dependent upon the difference of adjacent pressures and the corner coupling is only due to the off-diagonal tensor contributions. For $\tilde{\eta}=0$ diagonal dominance only occurs if off-diagonal tensor terms are zeroed and the 5 point stencil is recovered [15]. However for $\tilde{\eta}=0$, in addition to the framework, a symmetric positive definite discrete matrix can also be obtained by using the standard cell face harmonic mean for the diagonal tensor coefficients provided that

$$
\begin{gathered}
\left|K_{\alpha \beta_{i+1 / 2, j+1 / 2}}\right| \leq \sqrt{\min \left(K_{\alpha \alpha_{i+1 / 2, j}} \cdot K_{\alpha \alpha_{i+1 / 2, j+1}}\right)} x \\
\sqrt{\min \left(K_{\beta \beta_{i, j+1 / 2}}, K_{\left.\beta \beta_{i+1, j+1 / 2}\right)}\right.}
\end{gathered}
$$

[15] therefore it is only necessary to derive an appropriate homogenization for the off-diagonal terms of the tensor satisfying $\mathrm{Eq} 7.1$.

In summary : The general finite volume framework embodies many schemes in families on dual grids, including the Galerkin method and the mixed method. Further aspects concerning the quadrature are presented in $[15,16]$, where it is shown that the difference between family elementwise fluxes is no greater than $\alpha O(h)$ (where $\alpha$ is proportional to the cross derivative and $h$ is the element size), and that scheme control volume fluxes (and discretization error) differ by $\mathrm{O}\left(\mathrm{h}^{2}\right)$, providing an indication of relative convergence.

$h$-Adaptive Grids : In addition to regular grids, discretization at an h-adaptive grid interface requires particular care $[18,19]$. The above approach provides an extension of [18] for cell" centered full tensor discretization on non-orthogonal h-adaptive grids, Fig 5 illustrates two interface element mappings via renormalization.

\section{RESULTS}

The importance of modeling cross terms due to grid effects is illustrated in Fig 6, where (quarter 5-spot) miscible displacement is modelled using a higher order Godunov scheme [19] coupled with a 5 point operator on a non- orthogonal grid (N-O), Fig 6d shows a large grid induced error. Using the higher order scheme with the 9 point pressure equation scheme of section 6 removes the error Fig 6c.

The new renormalization operator has been demonstrated to provide increased accuracy compared to current techniques [14]. A comparison of the flux continuous cell centered 9 point scheme of [4] (labelled FC) with exact homogenization versus the cell centered 9 point scheme of section 6 with coefficients defined by the renormalization operator (approximate homogenization with assumed boundary conditions and discretization, and labelled RFC) is shown for two full tensor cases with $\mathrm{Kx}=\mathrm{Ky}=1, \mathrm{Kxy}$ $=\mathrm{Kyx}=-1 / 2$.

The first case is based on a modified Stones problem with 4 injectors and 1 producer and two shale permeability barriers aligned with the $\mathrm{x}$ axis as indicated in Fig 7. The pressure fields are in close agreement on a cartesian grid. The grid is adapted to the solution via an equidistribution technique [20] and improved resolution is obtained, particulary at the producer below the shale barrier. The effect is seen by both schemes (Fig $7 \mathrm{~d}, \mathrm{e}$ ) indicating that the new scheme exhibits consistent behaviour on a non-orthogonal grid.

The second case (Fig 8) has 4 injectors (at domain corners) and 1 producer (at domain center) and an array of 8 shale barriers. In this case 4 shales lie within a cross aligned with the $x$ and $y$ axes, while the remaining 4 shales are angled towards the middle of the domain providing a further non-orthogonality test. The grid is comprised of algebraic multiblocks e.g. $[21,22]$. This case provides a test of renormalization for both strong physical and grid non-orthogonality full tensor effects. The pressure field results again indicate good agreement Fig $8 \mathrm{c}$,d. The simpler scheme is able to detect all of the salient features, the importance of the full tensor renormalization coefficients is emphasized by a comparison using the arithmetic mean Fig $8 \mathrm{~b}$, which fails to detect the coefficient discontinuities.

\section{CONCLUSIONS}

A new renormalization operator based on the 9 point flux continuous scheme of [4] is presented and shown to be extremely effective when compared to the standard method of [14]. Preliminary results suggest that an effective permeability estimate depends upon the discretization and boundary conditions.

The new renormalization operator provides a natural means of mapping cell centered general tensor coefficients to cell vertices. The shift operator permits a transition to a general unifying finite volume framework for cell centered schemes applicable on non-orthogonal grids. The relationship between schemes in the framework is discussed for both of the dual discretizations (cell centered and vertex). With pressures located at the cell centers and coefficients at cell vertices, a discrete symmetric positive definite matrix is guaranteed for the entire family of 9 point in 2-D 19 or 27 in 3-D) schemes. Initial results demonstrate that important coefficient discontinuity induced pressure gradients are resolved by the new cell centered discretization. Many more numerical experiments await these new schemes. 
The techniques presented have great potential for reservoir simulation with important applications to upscaling and discretization.

Acknowledgments: US DOE (New generation reservoir simulation ACTI), US EPA (grant CR-821897-01-0) support is gratefully acknowledged.

\section{REFERENCES}

1 Thompson J.F., Warsi Z.U.A Mastin C.W. "Numerical Grid Generation: Foundations and Applications. North Holland New York 1985

2 King M.J., "Application and Analysis of Tensor Permeability to Crossbedded Reservoirs". SPE Paper 26118 (May 1993)

3 Durlofsky L.J., "Numerical Calculation of Equivalent Grid Block Permeability Tensors for Heterogeneous Media" Water Resources Research Vol 27 No. 5 pp 699-708 May 1991.

4 Edwards M.G. and C.F. Rogers "A Flux Continuous Scheme for the Full Tensor Pressure Equation" proceedings: 4th European Conference on the Mathematics of Oil Recovery. Eds. Christie et al, Norway, June 1994

5 Avatsmark A., T. Barkve, O. Boe and T. Mannseth, "Discretization on Non-Orthogonal, Curvilinear Grids for Multiphase Flow", Proceedings:4th European Conference on the Mathematics of Oil Recovery, Eds. Christie et al Norway, June 1994

6 Arbogast T., Wheeler M. F. and I. Yotov "Mixed Finite Elements for Elliptic Problems with Tensor Coefficients as Cell centered Finite Differences" to appear SIAM J. Numer. Anal. 1996

7 Edwards M.G. "Symmetric, Flux Continuous, Positive Definite Approximation of the Elliptic Full Tensor Pressure Equation in Local Conservation Form" SPE 2914713th SPE Reservoir Simulation Symposium, San Antonio, Texas, USA, pp 553 $562,12-15$ Feb 1995

8 Heinemann Z.E., Brand C.W. Munka M. and Chen Y.M. " Modeling Reservoir Geometry with Irregular Grids" SPE Res Eng 61991 pp 225 - 232

9 Avatsmark A., T. Barkve, O. Boe and T. Mannseth, "Discretization on Unstructured grids for Inhomogeneous, Anisotropic Media. Part 1: Derivation of Methods. Submitted SIAM J. Sci Comput.

10. K. Aziz and A. Settari, Petroleum reservoir simulation. Applied Science Publishers, London (1979)

11 Carey G. F.and Oden J. T., Finite Elements A Second Course Volume 2. Pub Prentice Hall
12 G.D. Smith Numerical Solution of Partial Differential Equations: Finite Difference Methods. Clarendon Press. Oxford 1985

13 Edwards M.G. and C.F. Rogers "A Cell Centered Finite Volume Scheme with Imposed Continuity for the General Pressure Equation" In revision 1996

14. King P.R., The use of renormalization for the calculating effective permeability. Transport in Porous Media 4, 37-58(1989)

15 Edwards M.G. "Finite Volume and Finite Element Discretisation of the Full Tensor Pressure Equation" In revision 1996

16 Edwards M.G. "Interaction of Accuracy and Cross Flow in Reservoir Simulation" in preparation

17 Arbogast T., Keenan P.T., Wheeler M. F. and I. Yotov "Logically Rectangular Mixed Methods fro Darcy Flow on General Geometry" SPE 2909913th SPE Reservoir Simulation Symposium, San Antonio, Texas, USA, pp 51 - 59, Feb 1995

18 Edwards M.G. "Elimination of Adaptive Grid Interface Errors in the Discrete Cell Centered Pressure Equation" To Appear: J. Comp. Phys

19 Edwards M.G. "A Higher Order Godunov Scheme Coupled With Dynamic Local Grid Refinement for Flow In a Porous Medium" To Appear: Comput. Meths. Appl. Mech. Engrg

20 Edwards M.G. Oden J.T. and Demkowicz L. "An h-r Adaptive Approximate Riemann Solver for the Euler Equations in Two Dimensions" SIAM J. Sci. Stat. Comput. vol 14 No. 1, pp. 185-217 1993

21 Forcey C.R. Edwards M.G. and Carr M.P. "An Investigation into Grid Patching Techniques" Proceedings of Numerical Grid Generation Techniques NASA Conference Pub No. 2166, Oct $6-7,1980$

22 Weatherill N. P. and Forcey C.R. "Grid Generation and Flow Calculations for Complex Aircraft Geometries Using a Multiblock Scheme" AIAA $84-1665,1984$ 


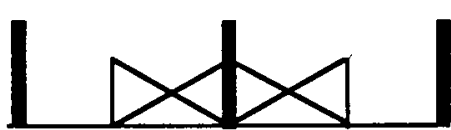

L $\quad F \quad R$

Fig 1 : Cell Centred and

Cell Face Pressures

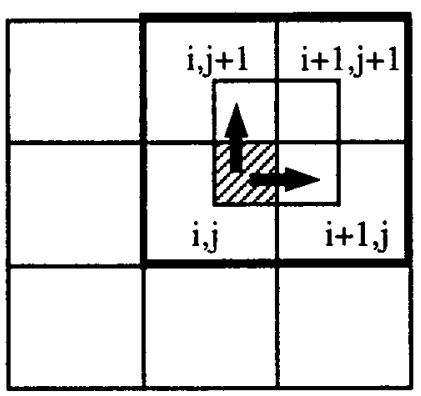

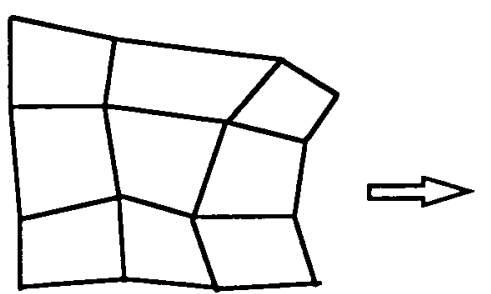

$\mathrm{x}, \mathrm{y}$

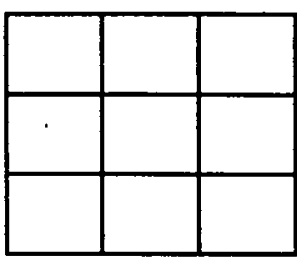

$\xi, \eta$

Fig 2 : Domain Mapping e.g. Thompson or Isoparametric per Multiblock
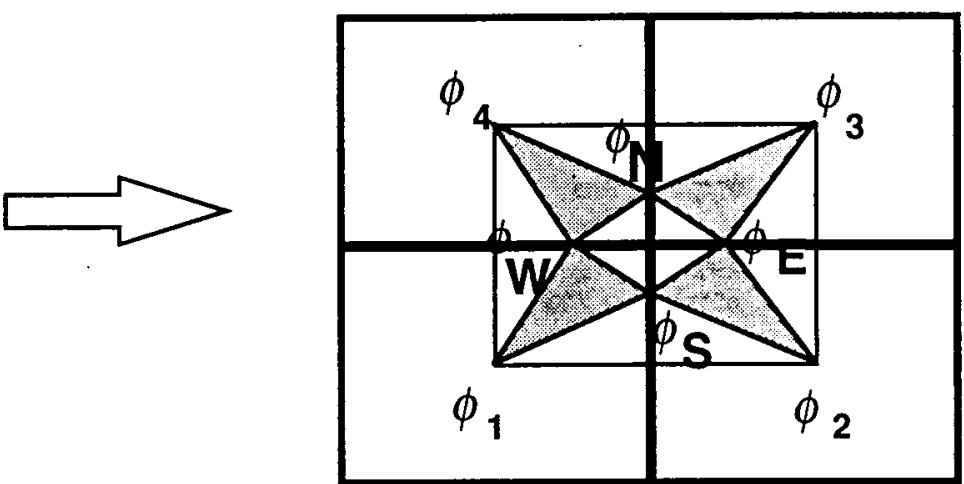

Fig 3 : Flux Continuous Tensor Scheme in

$\xi, \eta$
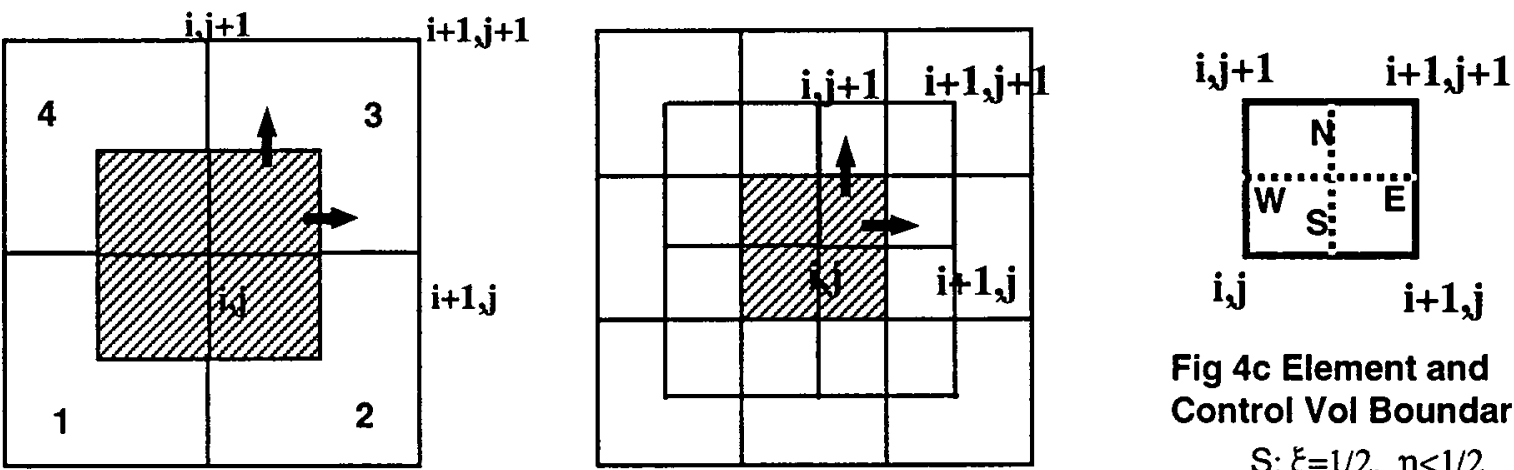

Fig 4c Element and Control Vol Boundaries $\mathrm{S}: \xi=1 / 2, \eta<1 / 2$

Fig 4a : Element $=$ Cell ; in $\xi, \eta \quad$ Fig $4 b$ : Element $=$ Union of 4 CellCenters Cell Vertex

Cell Centered
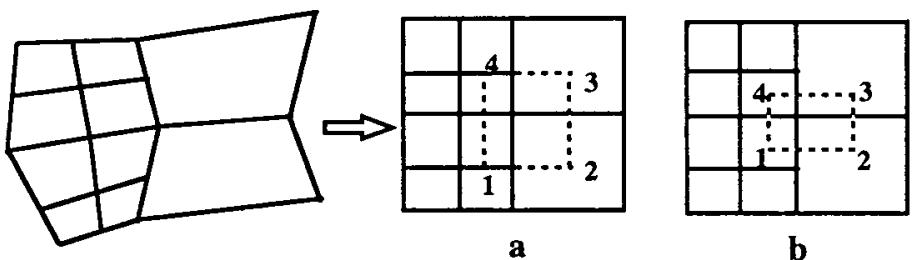

b

Fig 5 Renorm Map to Element (dashed line) 


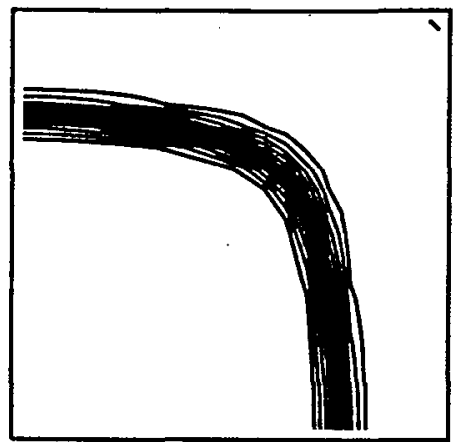

$6 a$ 20x20 Uniform Grid Solution

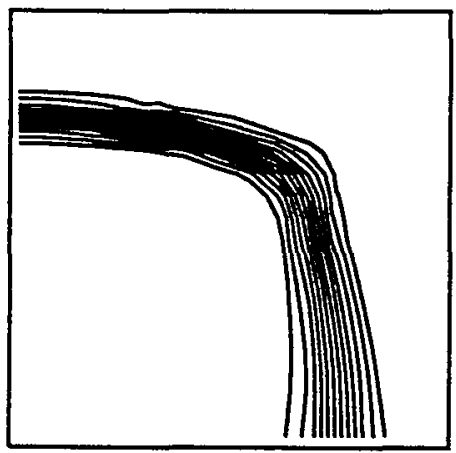

6c 9p t Full Tensor N-O Grid
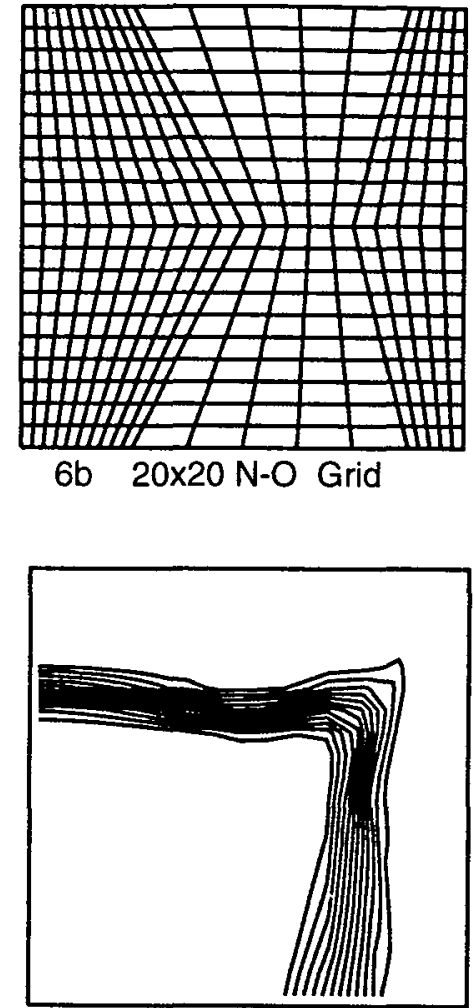

6d 5pt Diagonal Tensor N-O Grid

Higher Order Godunov Concentration Contours

Fig 6 9pt vs 5pt Pressure Eqn N-O Grid Test

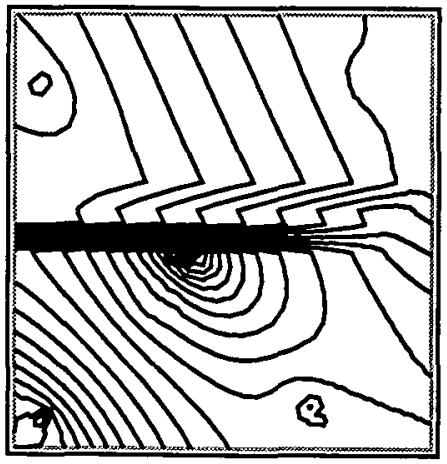

7a FC Cartesian Grid

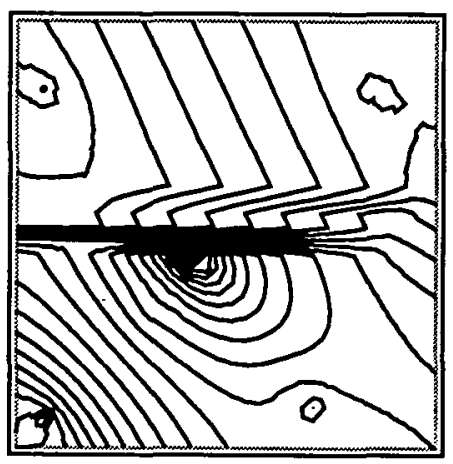

7b RFC Cartesian Grid

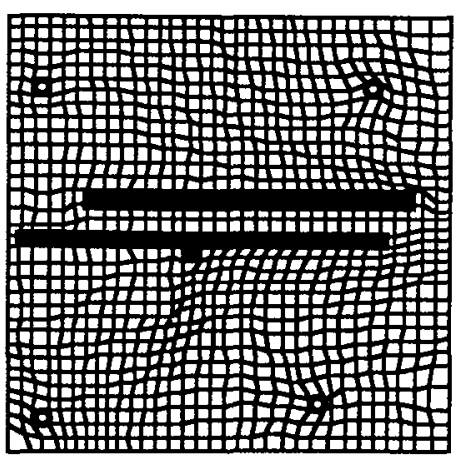

$32 \times 32$ N-O Redistributed Grid

2 shale permeability barriers

Fig 7 Pressure Contours 


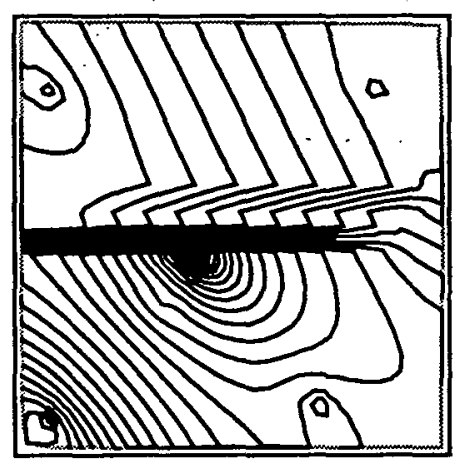

7d FC N-O

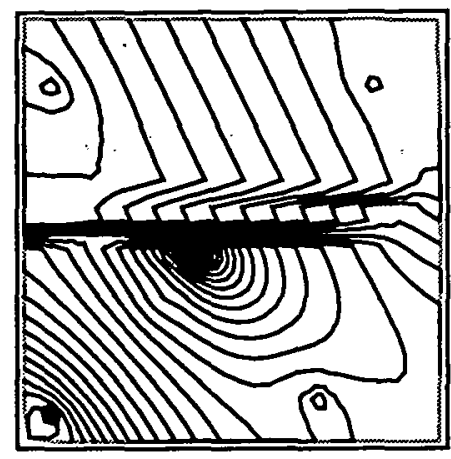

7e RFC (Renorm) N-O

Fig 7 Pressure Contours

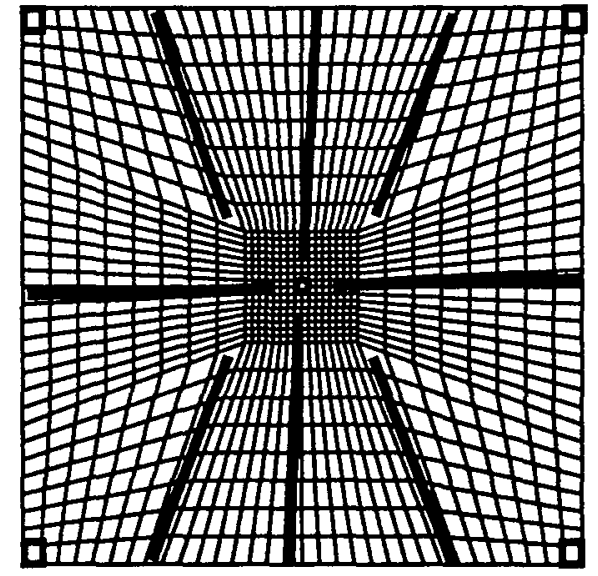

8 a $32 \times 32$ Grid with shales

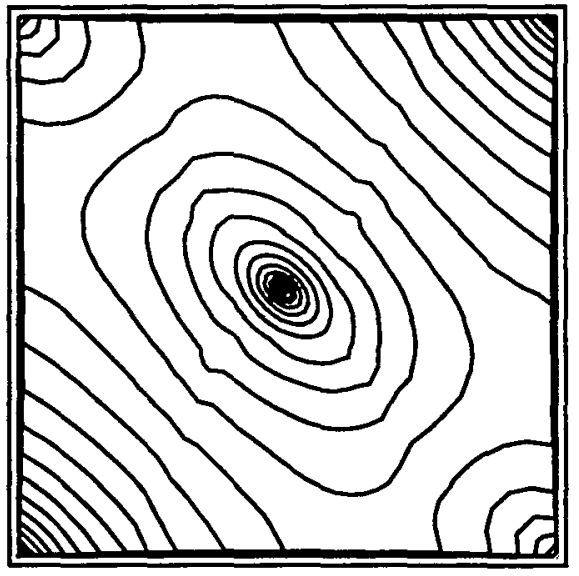

8b RFC Arithmetic Mean

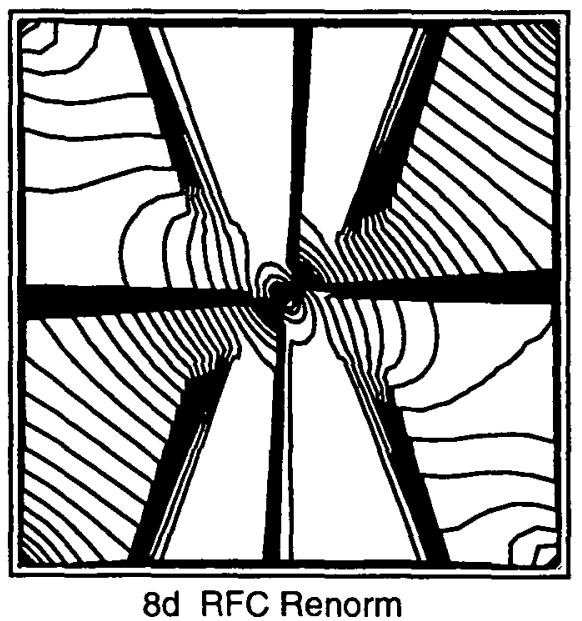

Fig 8: 8 Shales, Pressure Contours and Grid 